\title{
Fortschritt und Verdrängung - Ökologischer Fehlschluss und quantitative Revolution in der Geographie
}

\author{
Jan Kemper \\ Goethe-Universität Frankfurt, Institut für Humangeographie, Frankfurt, Germany \\ Correspondence: Jan Kemper (kemper@geo.uni-frankfurt.de)
}

Received: 19 December 2016 - Revised: 25 July 2017 - Accepted: 15 December 2017 - Published: 14 February 2018

\begin{abstract}
Kurzfassung. The quantitative revolution in the field of geography is commonly recalled as a story of scientific success, overcoming parochial, regionalist and ideographic modes of geography. This paper, however, suggests a different, yet parallel narrative. It reveals that the methodological reorientation towards statistical geography was a coping strategy, adopted by researchers from within and outside the discipline of geography in order to defend the analysis of ecological (i. e., spatially aggregated) data against the powerful critique of producing "ecological fallacies". Through emphasizing how the quantitative revolution was an expression of both modernizing and protective tendencies within the field of geography, the paper contributes to a more concrete understanding of what motivates methodological change in geography.
\end{abstract}

\section{Einleitung}

In seinem Diskussionsbeitrag Der Kieler Geographentag 1969 - Modernisierungsschub, Mythos, Paradigmenwandel oder vergessene Geschichte? (2016) kommt Peter Weichhart zu dem Ergebnis, das titelgebende „oder“ sei durch ein „und“ zu ersetzen (Weichhart, 2016:12). Der Beitrag lässt mindestens zwei weiterführende Lesarten der Begründung dieses Ergebnisses zu. In einer ersten möglichen Lesart ist das „und“ zwischen den Charakterisierungen Modernisierungsschub, Mythos, Paradigmenwandel sowie vergessene Geschichte additiv zu verstehen: Die Einschätzungen signalisieren unterschiedliche, aber sich ergänzende Erfahrungen mit dem in der Rede von „Kiel“ symbolisch repräsentierten Umbruchprozess im Feld der Geographie. In einer zweiten möglichen Lesart dagegen stehen diese Charakterisierungen weder gegen- noch nebeneinander, sondern verweisen als die Momente eines Prozesses der Wissenschaftsdynamik aufeinander: Das „und“ signalisiert, dass Modernisierungsschübe, Mythenbildungen, Paradigmenwandel und ein Vergessen der Geschichte zwar unterschiedliche, aber aufeinander verweisende Elemente von Wissenschaftsentwicklung beschreiben, die einen also nur durch die jeweils anderen vermittelt zu haben sind.
In den folgenden Ausführungen möchte ich diese zweite - und, wenn man so möchte, dialektische - Lesart aufgreifen und für ein Verständnis des als ,quantitative Revolution in der Geographie" (im Folgenden $\mathrm{qR}_{\mathrm{Geo}}$ ) bezeichneten Abschnitts der Geographieentwicklung fruchtbar machen. Mein Beitrag zielt darauf, in dem Umwälzungsprozess von Wissensbeständen und wissensgenerierenden Praktiken, der gemeinhin als $\mathrm{qR}_{\mathrm{Geo}}$ erinnert wird, die Verschränkung der Aspekte des Wissenschaftsfortschritts mit den Aspekten verzerrter Kommunikation (siehe dazu Habermas, 1982:445f.) sichtbar zu machen. Aus einer Perspektive, die Wissenschaftsprozesse vor allem als durch (disziplinübergreifend bzw. -diffundierend wirkende) Wissenschaftspraktiken strukturiert und Wissenschaftsentwicklung vor allem durch Auseinandersetzungen um die Angemessenheit von Forschungsstrategien und -methoden bestimmt sieht, konzentriere ich mich auf einen spezifischen Ausschnitt der raum- und sozialwissenschaftlichen Methodendiskussion: Ich diskutiere den 1950 erfolgten Nachweis des ökologischen Fehlschlusses in sozialwissenschaftlichen Forschungsarbeiten sowie die davon ausgehenden Folgen für eine in- wie außerhalb geographisch-disziplinärer Kontexte eingeübte konkrete wissenschaftliche Praxis. Verfolgt wird die These, dass das in der $\mathrm{qR}_{\mathrm{Geo}}$ zum Ausdruck gekommene verstärkte Interesse an quantitativen Forschungsmethoden und statistischen Verfah- 
ren auch eine Reaktion auf eine über die Kritik des ökologischen Fehlschlusses vermittelte, disziplinübergreifend wirksame Legitimationskrise des methodisch-empirischen Arbeitens mit raumbezogenen Aggregatdaten gewesen ist. Nicht zuletzt für den Zweck, diese Krisensituation zu bewältigen, ist nicht nur, aber eben auch in der Disziplin Geographie daran gegangen worden, den methodisch-statistischen Apparat zu modernisieren.

Von einem ökologischen Fehlschluss (ecological fallacy) wird in den Raum- und Sozialwissenschaften üblicherweise dann gesprochen, wenn von Zusammenhangsbehauptungen aus, die mit Hilfe des statistischen Konzepts der Korrelation und auf der Grundlage von Raum- bzw. Gebietseinheiten gewonnen wurden, auf das Handeln, Verhalten oder die Eigenschaften von Individuen (bzw. von mit bestimmten Merkmalen von Individuen assoziierten sozialen Gruppen) geschlussfolgert wird, etwa nach dem Muster: ,if countries with more Protestants have higher suicide rates, then Protestants must be more likely to commit suicide; if countries with more fat in the diet have higher rates of breast cancer, then women who eat fatty foods must be more likely to get breast cancer." (Freedman, 2001:4028) Solche Schlussfolgerungen sind nicht deshalb problematisch, weil sie eventuell inhaltlich falsch sind. Die Fehlleistung erstreckt sich vielmehr auf einen Irrtum im Gebrauch von Schlussregeln: Den Schlussfolgerungen fehlt eine gültiges Verfahren für das Schließen von A auf B (vgl. Haas, 2013).

Dass solche Schlussfolgerungen durch das methodische Verfahren der statistischen Korrelationsanalyse nicht gedeckt sind, solange raumbezogene Aggregatdaten das Untersuchungsmaterial geben, wurde durch den Mathematiker und Soziologen William S. Robinson in seinem vielzitierten Aufsatz Ecological Correlations and the Behavior of Individuals (1950) bewiesen. Der Beweis hatte erhebliche wissenschaftspraktische Konsequenzen, darin kommen auf das Problem des ökologischen Schließens konzentrierte Beiträge (Achen und Shively, 1995; Firebaugh, 2001; King, 1997) mit disziplinär orientierten Beiträgen etwa aus der politologischen Wahlforschung (Roth, 2008), der Epidemiologie (Subramanian et al., 2009) oder der Demographie (Voss, 2007) überein. Denn der Beweis legte nahe, zukünftig allein eine mit Survey- bzw. Paneldaten (also mit aus Befragungen von Einzelpersonen resultierenden Daten) arbeitende Methode als wissenschaftlich gültige Arbeitsweise anzusehen. Allen Forschungsansätzen der Soziologie und Geographie, der Geschichts- und Politikwissenschaften aber, die in ihrer empirischen Forschungsarbeit auf raumbezogene Aggregatdaten zurückgreifen (müssen), drohte vor diesem Hintergrund ihre wissenschaftliche Marginalisierung.

Dieser Rekurs auf einen wichtigen Einschnitt in der Anwendung statistischer Kalküle und auf dessen wissenschaftspraktische Folgen zielt nicht darauf, das in Debatten um den Charakter und Stellenwert der $\mathrm{qR}_{\mathrm{Geo}}$ deutlich werdende Interesse an einer (selbst-)reflexiven geographischen Wissensproduktion abzubrechen sowie darauf gerichtete For- derungen nach einer „unschärfere[n] Erzählung“ (Michel, 2014:301) von Fachgeschichte zugunsten einer manifesten Geschichte von Geographieentwicklung (erneut) zu unterlaufen. Im Gegenteil soll mit den folgenden Ausführungen dazu beigetragen werden, latente Inhalte des zur $\mathrm{qR}_{\mathrm{Geo}}$ verdichteten Wissenschaftsprozesses wieder freilegen zu können.

Mit dieser Absicht wird zugleich angeknüpft an das auch in der Geographie gewachsene Interesse für die Wirkmächtigkeit statistischer Kalküle in der Konstruktion und Bearbeitung sozialer Wirklichkeit (Barnes und Hannah, 2001; Sheppard, 2001, 2014). Der Beitrag folgt der Aufforderung, Methodendiskussionen über statistische Prozeduren und die Konzeptgeschichte statistischer Verfahren nicht allein den Statistikern zu überlassen, sondern sie selbst zum Gegenstand wissenschaftstheoretischer und -soziologischer Reflexion zu machen (vgl. Barnes und Hannah, 2001:379-380). Dabei reicht es nicht aus, Umbrüche in wissenschaftlichen Arbeitsweisen und Neuorientierungen in methodischen Verfahren vor dem Hintergrund wissenschaftsexterner Einflussmomente auf den Wissenschaftsprozess in ihren historischsozialen Kontexten zu situieren und von daher zu erklären. Auch die Rekonstruktion der wissenschaftsinternen, epistemischen Auseinandersetzungen über die wissenschaftliche Legitimität ihrer Ansätze und Methoden bleibt notwendig gerade für ein konkurrenz- und konfliktorientiertes Verständnis von Wissenschaftsgeschichte, das gegenüber gradlinig und widerspruchsfrei ausfallenden Erzählungen misstrauisch ist. Diese Notwendigkeit wird im Folgenden mit Blick auf die Geschichte der Entwicklung und des Einsatzes statistischer Konzepte und Verfahren deutlich. Denn erst in den wissenschaftsinternen Auseinandersetzungen wird mitentschieden, welche Konzepte und Verfahren noch als taugliche Produktionsmittel für die Produktion wissenschaftlichen Wissens gelten dürfen und welche abgeschrieben werden müssen.

Ausgangspunkt der folgenden Ausführungen ist daher die von Thomas S. Kuhn in Die Struktur wissenschaftlicher Revolutionen (2003; zuerst The Structure of Scientific Revolutions, 1962) aufgeworfene Frage nach den wissenschaftsinternen Möglichkeitsbedingungen wissenschaftlicher Revolutionen. Darauf bezogen erfolgt eine Analyse der Metaphorik der Revolution, wie sie den Klassiker Quantitative Revolution und theoretische Geographie (1970; zuerst The Quantitative Revolution and Theoretical Geography, 1963) des Geographen Ian Burton durchzieht. An diesem Beitrag wird eine Leerstelle im tradierten Rechtfertigungszusammenhang der $\mathrm{qR}_{\mathrm{Geo}}$ in Hinblick auf die Gelegenheitsstrukturen der $\mathrm{qR}_{\mathrm{Geo}}$ identifiziert (Kap. 2). Dann wird an den Vorschlägen zu einer Statistical Geography (Duncan et al., 1961) verfolgt, dass deren Formulierung als ein Methodenangebot an die Sozialwissenschaften wesentlich die Kritik des ökologischen Fehlschlusses an einem methodisch-empirischen Arbeiten mit Raumaggregatdaten reflektiert (Kap. 3). Anschließend werden die statistisch informierten Formen der Krisenbewälti- 
gung untersucht, mit denen es in- wie außerhalb der Disziplin Geographie ermöglicht wurde, der mit dem Nachweis des ökologischen Fehlschlusses gesetzten Herausforderung für ein empirisches Arbeiten mit raumbezogenen Aggregatdaten zu begegnen (Kap. 4). Abschließend wird das Verhältnis von Fortschritt und Verdrängung resümiert (Kap. 5).

\section{Wissenschaftliche Revolutionen und ihre Voraussetzungen}

\subsection{Die quantitative Revolution in der Geographie}

Anfang der 1960er Jahre zeigte sich der Geograph Ian Burton überzeugt, Zeuge und Anwalt einer epochemachenden Umwälzung in den Grundlagen seines Faches gewesen zu sein. „Während des letzten Jahrzehnts“, so sein Rückblick in Quantitative Revolution und theoretische Geographie (1970; zuerst The Quantitative Revolution and Theoretical Geography, 1963), habe „die Geographie einen radikalen Wandel von Geist und Zielsetzung erlebt, der am besten mit dem Ausdruck 'quantitative Revolution' umschrieben wird.“ (Burton, 1970:95) Burton trug nach: Die quantitative Revolution ,begann [...] in der Geographie erst in den späten vierziger oder frühen fünfziger Jahren; sie erreichte ihren Höhepunkt im Zeitraum von 1957 bis 1960 und ist nun vorüber.“ (Burton, 1970:97) An die Stelle des revolutionären Umbruchs sei jetzt - zu Beginn der 1960er Jahre - eine Phase der Konsolidierung getreten. Die ehemals ,revolutionären Ideen“ seien erfolgreich in einen „Bestandteil konventioneller Weisheit" (Burton, 1970:97) verwandelt, die Revolutionäre von einst „Teil des geographischen 'Establishments' und ihre Arbeit ein anerkannter und hochbewerteter Bestandteil des Fachs“ (Burton, 1970:99) geworden.

Als Ende der 1960er, Anfang der 1970er Jahre der Anschluss der deutschsprachigen geographischen Forschung an die quantitative „Bewegung“ (Burton, 1970:95) der englischsprachigen geographischen Forschungswelt begann (vgl. dazu etwa Giese, 1975:5), war Burtons Interpretation der damals jüngsten Geographiegeschichte als die Geschichte einer siegreichen wissenschaftlichen Revolution offenbar brauchbar für eine Rechtfertigung und Durchsetzung dieser Anschlussabsichten. Sein Aufsatz findet sich übersetzt in einer der damaligen „Schlüsselpublikationen“ (Weichhart, 2016:8), in dem heute klassisch anmutenden, von Dietrich Bartels herausgegebenen Sammelband Wirtschafts- und Sozialgeographie (1970). Dort wird über den Beitrag von Burton (und über weitere ins Deutsche übersetzte Beiträge) die Vorstellung einer ebenso dringlichen wie international bereits gelingenden nachholenden Modernisierung der Geographie vermittelt. Diese Modernisierung war für Burton und andere von der Aneignung quantitativer Methoden und von der Übernahme des ihnen entsprechenden Wissenschaftsverständnisses getragen: Aus „Unzufriedenheit mit der idiographischen Geographie“ (Burton, 1970:101) heraus habe eine nachwachsende Wissenschaftsgeneration die Beobach- tung und Beschreibung von raumbezogenen Regelmäßigkeiten und dafür geeignete komplexe statistische Verfahren Burton selbst denkt dabei vor allem an die Konzepte der Korrelation und Regression sowie an die probalistische Orientierung der Prüf- und Teststatistik - an die Stelle der Betonung und Untersuchung des Einzigartigen treten lassen. Die idiographische Orientierung der Geographie sei so einer stärker hypothesenprüfenden, theorie- und modellbezogenen Wissenschaftspraxis gewichen. Die quantitative Neuorientierung der Geographie habe damit erfolgreich ihr Ziel erlangt, die „Geographie wissenschaftlicher zu machen“ (Burton, 1970:101).

Die Kategorienwahl und die Verlaufsbeschreibung vermitteln zumindest auf einen ersten Blick den Eindruck, dass Burtons Darstellung der $\mathrm{qR}_{\mathrm{Geo}}$ stark von dem Schema der Wissenschaftsentwicklung beeinflusst wurde, das ein Jahr zuvor von Thomas S. Kuhn entfaltet worden ist. Wie Kuhn in Die Struktur wissenschaftlicher Revolutionen (2003; zuerst The Structure of Scientific Revolutions, 1962), greift auch Burton für eine Beschreibung von Veränderungen wissenschaftlichen Wissens auf die Vorstellung eines revolutionären Bruchs mit einer etablierten Wissensordnung sowie deren Ersetzung durch neue Forschungsorientierungen und -methoden zurück. Wie Kuhn stellt Burton deshalb die Durchsetzung der im Namen einer wissenschaftlichen Revolution betriebenen neuen, ,quantitativ“ bestimmten geographischen Wissensordnung als einen „Kampf“ dar, der mit „Behauptungen und Gegenbehauptungen, Angriffen und Gegenangriffen“ (Burton, 1970:99) zwischen den Revolutionären und einer „Opposition“ ausgetragen wird. Und wie Kuhn beschreibt Burton einen anschließenden Prozess der Normalisierung der revolutionären „neuen Ordnung“ (Burton, 1970:105): Sie erfährt ihre Institutionalisierung in den geographischen Curricula der Universitäten. Auf einen zweiten Blick allerdings wird deutlich, dass Burtons Skizze der $\mathrm{qR}_{\mathrm{Geo}}$ in entscheidenden Punkten von dem von Kuhn entfalteten Schema der Wissenschaftsentwicklung abweicht.

\subsection{Revolutionäre Situationen in der Wissenschaft}

Kuhn stieß sich bekanntlich an der Idee, einen wissenschaftlichen Durchbruch als ,ein einheitliches Ereignis, das einem bestimmten Menschen zu einer bestimmten Zeit und an einem bestimmten Ort zustößt“ zu behandeln, nämlich „so, als ob er etwas erblickt" (Kuhn, 1978:239). Dagegen machte Kuhn wissenschaftshistorische und -soziologische Einwände geltend. Sie zielten zum einen darauf hin, die heute allgemein akzeptierte wissenschaftssoziologische Grundposition zu fundamentieren, nach der die Produktion wissenschaftlichen Wissens ,sozial konstruiert“" sei, also aus Gesellschaftsabhängigkeiten heraus zu beschreiben und zu erklären ist. Zum anderen und vor allem aber liefen Kuhns Einwände darauf hinaus, ein dynamisches Modell der Wissenschaftsentwicklung zu entwerfen, das den Wandel wissenschaftlichen Wissens nicht primär mit dem Aufkommen und der 
Qualität „,neuer“ Forschungsansätze und -objekte in Verbindung bringt, sondern ihn vor allem aus dem Scheitern bereits etablierter forschungsleitender Grundannahmen und hergebrachter Gegenstandsbestimmungen heraus bestimmt.

Im Mittelpunkt dieses Modells steht das Konzept der Anomalie (vgl. dazu Kuhn, 2003:65ff.; siehe auch Weingart, 1976:33ff., 2003:41ff.). Es stellt in Kuhns Schema der Wissenschaftsentwicklung das entscheidende Bindeglied dar, mit dem zwischen den zwei anderen Basisannahmen Kuhns denen über eine etablierte, paradigmageleitete Forschungspraxis einerseits, dem Austausch dieser paradigmatischen Sichtweisen in „wissenschaftlichen Revolutionen“ andererseits - vermittelt werden kann. Anomalien sind für Kuhn solche Forschungsprobleme und -ereignisse, die gerade in der Verfolgung paradigmengeleiteter und deshalb ,normaler“ Forschung, also in einem gegebenen „System von Regeln“ (Kuhn, 2003:65) gesehen und formuliert werden können. Sie sind aber im Rahmen des gegeben Aussagensystems nicht oder nur unzureichend zu erklären bzw. zu ,„ösen“. Anomalien lassen demnach ein etabliertes Paradigma an seine Grenzen stoßen und letztlich scheitern: Sie sind die Irritationsmomente, die nicht mehr sinnvoll in gegebene „Orientierungskomplexe“ (Weingart, 1976:41) reintegriert werden können und somit zu Neuorientierungen zwingen.

\subsection{Die Leerstelle im tradierten Rechtfertigungszusammenhang der quantitativen Revolution}

Kuhn begnügte sich also nicht mit einer Beschreibung von Revolutionen auf dem Gebiet der Wissenschaft. Er fragte auch nach den Voraussetzungen für ihre Genese. Diese werden von Kuhn in krisenhaft anmutende Stockungen im Prozess paradigmageleiteter Wissensproduktion gesehen. Ohne Krise in der Produktion wissenschaftlichen Wissens keine revolutionäre Situation auf dem Gebiet einer Wissenschaft; ohne revolutionäre Situation aber keine wissenschaftliche Revolution.

In den Ausführungen von Burton nun hat dieser zentrale Aspekt des Kuhnschen Schemas der Wissenschaftsentwicklung offensichtlich keinen Widerhall gefunden. Burtons Schilderung der Geographieentwicklung in den 1950er und 1960er Jahren als die Geschichte einer wissenschaftlichen Revolution verzichtet nicht nur auf den für Kuhn zentralen Begriff des Paradigmas, sondern auch auf Kuhns Konzept der Anomalie. Ihm fehlt es deshalb an Vermittlungsschritten, die den Übergang von einer vorrevolutionären Geographie in den Prozess ihrer quantitativen Revolutionierung aus der Bestimmung einer revolutionären Situation aus der etablierten geographischen Wissenschaftspraxis selbst heraus verständlich machen könnten.

Stattdessen lässt Burton zum einen die „Quantifizierung“ der Geographie von außen kommend auf die Geographie übergreifen: Er schildert sie als ein „Resultat von Wirkungen nichtgeographischer Arbeiten auf die Geographie“ (Bur- ton, 1970:95), als „Invasion“ (Burton, 1970:98). Zum anderen fokussiert er auf die Motive und das Selbstverständnis der Trägergruppe der Revolution. Mit diesen Aspekten wird vielleicht deutlich, dass eine junge Wissenschaftsgeneration die Geographie paradigmatisch revolutionieren wollte und welche intellektuellen Ressourcen ihr dafür zur Verfügung standen. Offen hingegen bleibt, warum Anfang der 1950er Jahre ein nach Burtons Darstellung die Geographie dominierendes idiographisches Paradigma seine eigene Interpretationsund Erklärungskraft nicht mehr aufrechterhalten konnte. Die für eine Erklärung wissenschaftlicher Revolutionen auf dem Gebiet der Geographie zentrale Frage, was die bis dahin dominierende geographische Forschungspraxis in der Verfolgung ihrer eigenen Forschungs- oder Argumentationsweise auf Widersprüche und an Grenzen stoßen und somit dermaßen in eine Krise stürzen ließ, dass eine Umwälzung der wissenschaftlichen Arbeitsgrundlagen attraktiv erschien - also eine revolutionäre Situation auf dem Feld der Geographie erst bedingte - wird in Burtons Darstellung der $\mathrm{qR}_{\mathrm{Geo}}$ nicht aufgeworfen, geschweige denn beantwortet. Die Behauptung einer revolutionären Umwälzung geographischer Forschung soll offensichtlich ohne eine Erinnerung an deren Gelegenheitsstrukturen auskommen können, statt dessen als die mutige Tat wissenschaftlicher Revolutionäre gegenüber einem als wissenschaftlich ungenügsam definierten Status quo im Gedächtnis bleiben.

\section{Anomalie und Krise}

\subsection{Statistische Geographie als Methode}

Im Folgenden wird daran gegangen, in Anschluss an Kuhns Auffassungen von Wissenschaft und Wissenschaftsentwicklung eine anomalische Situation zu rekonstruieren, die eine nicht nur, aber auch auf dem Gebiet geographischer Wissensproduktion etablierte Wissenschaftspraxis hinterfragte und somit Anlass für ein gesteigertes Interesse an Diskussionen über quantitative Methoden und an Innovationen auf dem Gebiet der Statistik gab.

Eine Reflexion der Geographiegeschichte im Stil von Burton (1970) gibt, wie gesehen, für eine solche Rekonstruktion nicht viel her. Wissenschaftsentwicklung und -begründung, Genese und Legitimation der $\mathrm{qR}_{\mathrm{Geo}}$ erscheinen dort eng miteinander verknüpft und stützen sich gegenseitig: Weil eine quantitativ orientierte einer idiographischen Wissenschaftspraxis wissenschaftlich überlegen gewesen sei, soll das idiographische dem quantitativ orientierten Wissenschaftsverständnis in der Geographie gewichen sein; weil ein idiographisches einem quantitativ orientierten Wissenschaftsverständnis gewichen ist, zeige das den Wissenschaftsfortschritt der Geographie an. Dieses Rechtfertigungsmuster bleibt ganz dem Selbstverständnis erfolgreicher wissenschaftlicher Revolutionäre, ihren Überzeugungen von der eigenen Wissenschaftlichkeit und von der wissenschaftlichen Ungenügsamkeit des vormaligen Status quo verhaftet. Schon ganz aus 
der Perspektive eines durchgesetzten neuen Paradigmas und aus dessen Problemhorizonten heraus formuliert, ist die anomalische Situation offensichtlich gar nicht mehr präsent, aus der eine Neustrukturierung der Wissenschaftspraxis versprach, erfolgreich hinauszuführen.

Wie eine konkreter Antwortversuch auf eine Herausforderung dagegen liest sich noch die Abhandlung Statistical Geography: Problems in Analyzing Area Data (Duncan et al., 1961). Diese Veröffentlichung - darauf machte schon Bartels in seiner Aneignung „neuerer englischsprachiger Literatur“ zum Phänomen ,quantitative Geographie“ aufmerksam (vgl. Bartels, 1969:136) -, scheint die erste überhaupt zu sein, für die Bezüge auf ein neues, ,quantitatives“ Paradigma in geographisch orientierten Forschungsansätzen titelgebend sind; und dort schwingt schon im Untertitel auch noch eine Problemreferenz mit, die jeder Rechtfertigung einer neuen paradigmatischen Orientierung eigen sein muss.

Statistical Geography trägt in weiten Zügen den Charakter eines Lehrbuchs. Das Buch informiert über statistische Mittel bzw. quantitativ angelegte Methoden für raumbezogene Analysen, maßgeblich auf dem Terrain der Demographie und des Sozialen. Es wird im Vorwort als ein „,by-product of several years of research on areal problems" (Duncan et al., 1961:v) vorgestellt; ruht also auf den Forschungsarbeiten, die in den Jahren zuvor angefertigt worden sind.

Mit Blick auf die disziplinäre Einbettung des Werkes handelt es sich um eine Arbeit von Soziologen. Sein zentraler Autor, Otis D. Duncan, ist in den 1950er Jahren einer der führenden Vertreter des quantitativ orientierten Flügels bzw. der „3. Phase“ der Chicago School of Sociology (vgl. zu diesen Einordnungen Calhoun und VanAntwerpen, 2007:396-402). Doch schon der Titel des Buches und die darin anschaulich werdende diskursive Umbettung machen deutlich: Statistical Geography verweist ganz offensichtlich auf einen nicht-soziologischen Referenzrahmen. Folgt man Vorwort und Einführungskapitel, ist dieser neue Referenzrahmen wiederum ausschließlich methodisch zu verstehen. „Statistische Geographie“ wird als Kurzformel für die Lehre des richtigen Umgangs mit ,special features of areal data“ (Duncan et al., 1961:6) und davon ausgehende Schwierigkeiten eingeführt. Sie ist somit nicht als Anleitung für ein spezifisches, disziplingebundenes Geographie-Machen gemeint. „Statistische Geographie“ wird vielmehr als ein disziplinübergreifendes Angebot präsentiert: Sie wird für all diejenigen aufgeboten, die ,in such diverse subjects as economic geography, plant ecology, agricultural economics, and urban sociology" (Duncan et al., 1961:4) vor gleichen methodischen Herausforderungen im Umgang mit ,spatial and areal problems“ (Duncan et al., 1961:4) bzw. mit „,regional problems" (Duncan et al., 1961:11) stehen.

Dieses Angebot einer von engen disziplinären Bezügen entkoppelte, auf den Umgang mit areal data konzentrierten Methodenlehre wiederum wird durch einen ganz spezifischen Problemkomplex motiviert. Das Bedürfnis nach einer disziplinübergreifenden, statistisch-geographisch ausfal- lenden Forschungsmethode entspringt, ,analogous problems in making inferences from statistical data ordered by areal units" (Duncan et al., 1961:4). Neben der Frage nach validen Mitteln für eine Bestimmung der räumlichen Konzentration und Segregation von Bevölkerungsgruppen sieht Statistical Geography diese Problemlage vor allem durch die in Frage gestellte Gültigkeit von ,areal correlations“ als ein Mittel für Aussagen über ,individual relationships“ (Duncan et al., 1961:9) bestimmt - also durch die Kritik des ökologischen Fehlschlusses strukturiert.

\subsection{Der Nachweis des ökologischen Fehlschlusses}

Auf einen ökologischen Fehlschluss (ecological fallacy) sind die Sozialwissenschaften erstmals durch den Aufsatz Ecological Correlations and the Behavior of Individuals des Soziologen und Mathematikers William S. Robinson aufmerksam geworden, der 1950 in der American Sociological Review erschien. ${ }^{1}$ Diesem Beitrag verdanken die Sozialwissenschaften ihre (allerdings auch immer wieder verdrängte) Einsicht, dass Aussagen etwa über Beziehungen zwischen dem Wahlverhalten, der Devianz oder auch dem Krankheitsbild der Einzelnen einerseits, ihrem sozioökonomischen Status andererseits nicht zuverlässig aus solchen Kenntnissen abgeleitet werden können, die über Beziehungen zwischen diesen Merkmalen vorliegen, die aus in Raumausschnitten aggregierten Daten gewonnen wurden - also etwa auf eine Beziehung zwischen Wahlverhalten und sozioökonomischem Status verwiesen wird, die auf Grundlage einer Untersuchung von Parteianteilen und der Zusammensetzung der Sozialstruktur der Wählerschaft auf der Ebene der Stadtteile konstatiert werden kann. ${ }^{2}$

Der Nachweis der Unzulässigkeit dieser Ableitung gelang Robinson in einer Auseinandersetzung mit der Aussagekraft und des Verwendungszusammenhangs von Korrelationskoeffizienten. Er bewies, dass ein mit Hilfe des Konzepts der Korrelation festgehaltener statistischer Zusammenhang zwischen zwei Merkmalen, der für eine räumliche Aggregatebene festgehalten wurde, nicht sicher auf einen Zusammenhang

\footnotetext{
${ }^{1}$ Der Ausdruck ecological fallacy wird regelmäßig direkt mit Robinsons Artikel von 1950 verbunden; Robinson selbst hatte diesen Term allerdings noch nicht aufgebracht (vgl. Firebaugh 2001:4024; Subramanian 2009:343; Voss 2007:463). Robinson war, wie King (1997) festhält, zudem auch nicht der erste, der inhaltlich betrachtet auf das Problem des ökologischen Fehlschlusses gestoBen ist. Aber , ,...] for not until Robinson's (1950) article was the problem widely recognized and the quest for a valid method of making ecological inferences begun in earnest." (King 1997:4).

${ }^{2}$ So aber verfährt aktuell zum Beispiel eine Studie der Bertelsmann-Stiftung (Schäfer et al., 2013). Die Untersuchung möchte über die Beobachtung von statistischen Zusammenhängen zwischen Arbeitslosenquoten und Wahlbeteiligungsraten auf der Grundlage stadtteilbezogener Daten entlang der Bundestagswahl 2013 auf die soziale Selektivität des (Nicht-)Wählens aufmerksam machen.
} 
Tabelle 1. Korrelationskoeffizienten für ökologische und individuelle Korrelationen: Die Beispiele in Robinson (1950).

\begin{tabular}{lrr}
\hline Ebene & Beispiel A & Beispiel B \\
\cline { 2 - 3 } & $\begin{array}{rrr}\text { (,Illiterate“/ } \\
\text { „Negro“) }\end{array}$ & $\begin{array}{r}\text { (,Illiterate“/ } \\
\text { „Foreign-Born“) }\end{array}$ \\
\hline Divisions & 0.946 & -0.619 \\
States & 0.773 & -0.526 \\
Individual & 0.203 & 0.118 \\
\hline
\end{tabular}

zwischen diesen Merkmalen auf Individualebene zurückgeschlossen werden kann, weil sich dort ein ganz anderer statistischer Zusammenhang zeigen kann.

Wie sehr Korrelationskoeffizienten je nach der gewählten Beobachtungs- bzw. Datenerhebungsebene voneinander abweichen können, demonstrierte Robinson an zwei empirischen Beispielen. Er ging entlang der amtlichen ZensusDaten von 1930 sowohl auf der Ebene der neun ZensusBezirke („Divisions“), der Bundesstaaten sowie auf Individualebene zum einen der Frage nach, ob sich tatsächlich ein statistischer Zusammenhang in den Verteilungsmustern der Attribute „Illiterate“ und „Negro“ nachweisen lässt (Beispiel A). Zum anderen thematisierte er ebenfalls auf allen drei Untersuchungsebenen einen behaupteten Zusammenhang in den Verteilungsmustern der Attribute „Illiterate“ und „ForeignBorn“" (Beispiel B). ${ }^{3}$

Tabelle 1 hält Robinsons Ergebnisse für die beiden genannten Beispiele fest. Die Ergebnisse verdeutlichen erstens: Die Korrelationskoeffizienten variieren offensichtlich deutlich in Hinblick auf die Stärke des behaupteten statistischen Zusammenhangs. Für beide Beispiele lassen sich moderate bis sehr starke statistische Zusammenhänge für die Aggregatebenen „Divisions“ und „States“, aber nur schwache statistische Zusammenhänge auf der Individualebene festhalten. An Beispiel B wird zweitens deutlich: Sogar eine Verkehrung der Richtung der Zusammenhangsbeobachtungen ist möglich. Während die Ergebnisse für die Aggregatebenen einen gegenläufigen Zusammenhang zwischen den Merkmalen „Illiterate“ und „Foreign-Born“ suggerieren, wird auf Individualebene ein (allerdings schwacher) gleichläufiger $\mathrm{Zu}$ sammenhang deutlich. Diese Beobachtungen bleiben trotz einer 2011 erfolgten Überprüfung der Auswertungsschritte von Robinson, die auf eine Reihe von Unregelmäßigkeiten aufmerksam gemacht hat, gültig. Eine Wiederholung der Beweisführung von Robinson unter Vermeidung dieser Unre-

\footnotetext{
${ }^{3} \mathrm{Zu}$ gesellschaftlichen und wissenschaftsgeschichtlichen Hintergründen von Robinsons Problematisierung siehe die von Subramanian et al. (2009) gemachten Überlegungen sowie die daran anknüpfende, u. a. von Oakes (2009) artikulierte Kritik im International Journal of Epidemiology. Im Rahmen der dort geführten Debatte über den ökologischen Fehlschluss ist auch Robinsons Aufsatz von 1950 wieder abgedruckt worden (Robinson, 2009).
}

gelmäßigkeiten zeigt ähnliche Ergebnisse an (vgl. te Grotenhuis et al., 2011).

\subsection{Kritik und Disqualifizierung der Raumaggregatdatenanalyse}

Warum Robinsons Problematisierung des ökologischen Fehlschlusses im Rahmen der Statistical Geography als eine anzugehende Herausforderung begriffen wurde, liegt der Sache nach auf der Hand. Die Anwendung statistischer Verfahren stand und steht in der erkenntnistheoretischen Tradition einer realistisch ausfallenden Vorstellung von der Wirklichkeit und ihren Erkenntnismöglichkeiten (vgl. Schnapp et al., 2006:13). Bei aller (heute vielleicht mehr, damals sicherlich weniger) ausgeprägten Sensibilität für die Konstruktionsaspekte in der Prozesskette der Datenerhebung, -aufbereitung und -auswertung bleibt deren Anwendung selbst auf das Ziel bezogen, realitätstüchtige Abbilder der Wirklichkeit zu produzieren. Der Hinweis auf je nach Beobachtungs- bzw. Datenerhebungsebene variierende Korrelationskoeffizienten aber begrenzte deren Aussagekraft deutlich auf einen spezifischen, ausgewählten Zuschnitt dieser Wirklichkeit. Damit wurde konkret der Anspruch der raumbezogen und im sozialökologischen Paradigma arbeitenden Sozialwissenschaften zurückgewiesen, Erklärungen für soziale Tatsachen anbieten zu können; darüber hinaus aber mussten so Zusammenhangsaussagen (zunächst einmal) als tendenziell beliebige Aussagen erscheinen (vgl. Voss, 2007:463).

Diese Infragestellung damaliger Selbstgewissheiten griff vermutlich umso mehr, als dass Robinsons Kritik nicht von außen an einen etablierten methodischen Status quo herangetragen, sondern als interne Kritik statistischer Wissensproduktion entwickelt wurde. Seine Kritik rief also nicht - wie etwa in der Diskussion qualitativer vs. quantitativer Methoden - andere, den statistischen Verfahren externe Bezugspunkte auf, die dann gegen den Einsatz statistischer Kalküle gewendet wurden. Sondern sie bestritt gerade auf Grundlage der damals etablierten Prozeduren statistischen Arbeitens, also unter Voraussetzung und nach den Regeln der eingesetzten Verfahrenstechniken selbst die Gültigkeit der so produzierten Ergebnisse. Mit anderen, an Kuhns Wissenschaftstheorie orientierten Worten: Mit der internen Kritik des ökologischen Fehlschlusses verwies Robinson auf eine Anomalie. Sie tritt unter den Bedingungen der eingespielten Forschungspraxis hervor, ohne aber von dieser sinnvoll reintegriert werden zu können. Von der anomalischen Situation geht daher die Aufforderung aus, durch einen ,Wechsel der Ausrüstung“ (Kuhn, 2003:89) bzw. durch einen „Neuaufbau des Gebiets auf neuen Grundlagen“ (Kuhn, 2003:98), also durch eine wissenschaftliche Revolution aus ihr herauszuführen.

Dieser Wechsel der methodischen Ausrüstung wird von Robinson allerdings nicht nur indirekt nahegelegt, sondern auch offensiv propagiert. Mit polemischen Zuspitzungen führt er die Erkenntniseffekte seiner Kritik zu ihren wissen- 
schaftspraktischen Konsequenzen. Robinson versichert sich und seinem Lesepublikum (1) der definitiven Untauglichkeit der räumlich orientierten Aggregatdatenanalyse für Rückschlüsse auf die Individualebene (vgl. Robinson, 1950:357). Die übersehene Untauglichkeit aber (2) ,throws serious doubt upon the validity of a number of important studies made in recent years" (Robinson, 1950:357). Robinson zieht in seinem Artikel prominente Werke der amerikanischen Sozialwissenschaften, die seit den 1920er Jahren vor allem unter sozialökologischem Vorzeichen entstanden sind - so etwa die Studie Delinquency Areas: A Study of the Geographic Distribution of School Truants, Juvenile Delinquents, and Adult Offenders in Chicago (Shaw et al., 1929) - als Belege für ein fehlgehendes ökologisches Schließen an. Die Arbeiten werden so dezidiert als unwissenschaftlich ausgewiesen. Diese Entwertung von Wissensbeständen wiederum ist von Robinson mit (3) der expliziten Aufforderung zu einer methodischen Neuorientierung verbunden worden. Sein Artikel schließt mit einem Plädoyer, das eine Abkehr von den ,,meaningless correlations" der mit Gebietseinheiten operierenden Aggregatdatenanalyse, statt dessen die Hinwendung zu einer Untersuchung von „meaningful correlations between the properties of individuals" (Robinson, 1950:357) qua Individualdaten verlangt, wenn Aussagen über das Handeln und das Verhalten von Einzelnen gemacht werden sollen.

Mit den Absichten von Duncan et al. (1961), Möglichkeiten und Chancen für eine „statistische Geographie“ auszuloten, ist vielleicht am deutlichsten auf Robinsons Kritik am ökologischen Fehlschluss und auf die davon ausgehende Provokation für eine mit Raumaggregatdaten arbeitende methodisch-empirische Arbeitsweise reagiert worden. Doch verunsichert von diesem Problemkomplex wirkt auch schon eines der allerersten Dokumente, das nach Barnes (1998:216) mit dem $\mathrm{qR}_{\mathrm{Geo}}$ genannten Umbruchsprozess verbunden werden kann. Der Bericht The Measurement of Association in Industrial Geography (McCarty et al., 1956) sollte grundsätzlich die Übertragbarkeit von ,certain statistical techniques to problems employing an areal frame of reference“ (McCarty et al., 1956:iv) demonstrieren, sah aber zugleich „deficiencies in many of these approaches" (McCarty et al., 1956:iv). Konkreter werden ,problems arising in connection with the measurement of geographic association“ (McCarty et al., 1956:1) angesprochen. Solche Probleme werden unter anderem in Hinblick auf den Themenpunkt „Scale“ ausgemacht. Wie ein frühes Echo auf Robinsons Kritik am ökologischen Fehlschluss liest sich deshalb die dort artikulierte Einsicht, ,that conclusions derived from studies made at one scale should not be expected to apply to problems whose data are expressed at other scales. Every change in scale will bring about the statement of a new problem, and there is no basis for presuming that associations existing at one scale will also exist at another." (McCarty et al., 1956:160; die explizite Verbindung von dort zum Problemkreis des ökologischen Schließens ziehen Duncan et al., 1961:9 sowie Thomas und Anderson, 1965:492)
Solche Einsichten in die Begrenztheit der Generalisierbarkeit und damit Aussagekraft von mit Raumaggregatdaten gewonnenen Forschungsergebnissen haben vermutlich mit dazu beigetragen, dass die in den 1950er Jahren vollzogene Aneignung von neuen statistischen Verfahren, insbesondere der Korrelation und der Regression (siehe dazu ausführlich Barnes, 1998:215ff.) auch innerhalb geographisch-disziplinär verstandener Kontexte nicht allein als ,an unmixed blessing“ (Thomas und Anderson, 1965:492) eingeschätzt wurde, sondern auch als ein Irritationsprozess begann, der nach Bewältigungsstrategien verlangte. Denn ,their adoption also has presented the geographer with methodological problems which were not important to him or which he did not clearly recognize when his research was conducted at a less precise level“" (Thomas und Anderson, 1965:492).

\section{Modernisierungsdruck und Krisenreaktionen}

\subsection{Die Legitimationskrise der Raumaggregatdatenanalyse}

In heutigen Kommentaren (Achen und Shively, 1995; Firebaugh, 2001; King, 1997; Voss, 2007) herrscht Einigkeit darüber, Robinsons Verknüpfung von Kritik und Polemik eine durchschlagende Wirkung auf damals etablierte sozialwissenschaftliche Forschungsfelder und -praktiken zuzusprechen. Demnach marginalisierte Robinsons Problematisierung des ökologischen Fehlschlusses nicht nur grundlegend eine der bis dahin zentralen methodischen Vorgehensweisen und die daran geknüpften Forschungsarbeiten. Er zwang somit auch ganze Forschungsfelder und -ansätze zur Einstellung ihrer bisherigen und nötigte zum Aufbau neuer Forschungsorientierungen. Denn in dem Maße, wie an Stelle der nun für die wissenschaftliche Aussagenproduktion weitgehend disqualifiziert anmutenden Aggregatdatenanalyse mehr und mehr die statistische Analyse von Individualdaten als das angemessene Mittel der Erkenntnisgewinnung trat, verschoben sich notgedrungen auch Forschungsthemen, -fragestellungen und -instrumente. Mit der Konzentration auf die Auswertung von Daten auf Individualebene rückte zum einen das Instrument des Surveys immer stärker in den Vordergrund. Damit aber wurden solche Forschungsthemen und -fragestellungen favorisiert, die sich qua Umfrage angehen und beantworten lassen - also solche, die über eine Erhebung individueller Attribute und subjektiver Einstellungen erschlossen werden können. Darüber hinaus erfordert eine Verallgemeinerung von Interviewergebnissen methodisch betrachtet ihre Organisation als Zufallsstichprobe. Das wiederum führt zu einer Datengrundlage, deren sinnvolle Auswertung eine Beherrschung der auf wahrscheinlichkeitstheoretischen Grundlagen beruhenden Verfahren der Prüf- und Teststatistik voraussetzt.

Diese inhaltlichen und methodischen Aspekte zeigen zum anderen an, welche Forschungswege bzw. Wissenschaftszweige durch den Nachweis des ökologischen Fehlschlus- 
ses mit starken Legitimationsproblemen konfrontiert waren und unter einen erheblichen Modernisierungsdruck gerieten. Der nun dominant werdende „focus on national surveys with random interviews of isolated individuals“ (King, 1997:5) als Maßstab für anzuerkennende Erkenntnisansprüche führte all jene sozialwissenschaftlichen Forschungsrichtungen in eine Krise, die ihre Forschungstätigkeit nur schlecht auf personenorientierte, gegenwartsbezogene Umfragen stützen können - nach Firebaugh (2001) oder King (1997) vor allem geographisch und historisch ausgerichtete Forschungszweige; nach Voss (2007) vor allem die am sozialökologischen Paradigma orientierte sozialwissenschaftliche Forschung. Wenn diese nicht mangels Alternativen in der bisherigen Form, aber ,under a cloud of great suspicion“ (King, 1997:5) weiterarbeiten wollten, standen sie vor der Entscheidungsherausforderung, eine räumlich orientierte, mit Gebietseinheiten operierende und auf die Aggregatdatenanalyse fokussierte Arbeitsweise entweder aufzugeben oder den bisherigen methodischen Status quo mit neuen Mitteln und auf neuen Wegen verteidigen zu müssen.

\subsection{Die Formen des Krisenmanagements}

Wenn richtig ist, dass mit Robinsons Nachweis des ökologischen Fehlschlusses mit Anfang der 1950er Jahre ein Festhalten an räumlich orientierten, mit Gebietseinheiten operierenden und auf die Aggregatdatenanalyse fokussierten Forschungsansätzen in- wie außerhalb der Geographie problematisch wurde, liegt es nahe, von dort aus hauptsächlich zwei Reaktionsweisen zu erwarten. Sie können in Anlehnung an die von Albert O. Hirschman mit Abwanderung und Widerspruch (1974; zuerst Exit, Voice, and Loyality 1970) in politisch-ökonomische und politiktheoretische Diskurse eingeführte Unterscheidung zwischen Handlungsoptionen in Krisensituationen als Reaktionen der „Abwanderung“ (bzw. von „Exit") und des „Widerspruchs“ (bzw. von „Voice“) charakterisiert werden: Abwanderungs- bzw. ExitReaktionen laufen auf die Abkehr von einer methodischempirischen Arbeitsweise mit raumbezogenen Aggregatdaten heraus; über die Organisation von Widerspruch dagegen wird versucht, Formen der Selbstbehauptung dieser Wissenschaftspraxis gegen ihre drohende Entwertung zu entwickeln.

Mit diesen Vorüberlegungen lassen sich vier Verarbeitungsstrategien der Kritik des ökologischen Fehlschlusses skizzieren: Neben (1.) dem Verzicht auf die Methode der Raumaggregatdatenanalyse lassen sich in Hinblick auf (2.) ihre Verteidigungsversuche (a) eine offensiv verfochtene Rehabilitation des ökologischen Schließens, (b) eine defensiv orientierte Kooption der Kritik von Robinson und (c) eine Rückzugsbewegung festhalten. Entscheidend an allen vier Verarbeitungsstrategien aber ist: Sie kamen nicht ohne Innovationen auf dem Gebiet der Statistik aus.
1. Abwanderungs-bzw. Exit-Reaktionen forcieren den forschungspraktischen Ausstieg aus dem wissenschaftlichen Arbeiten mit raumbezogenen Aggregatdaten. Die Krise dieser Wissenschaftspraxis wird über eine Kapitulation vor der Kritik, also durch eine Absage an eine raumbezogene Aggregatdatenanalyse und durch Umstellung auf die Survey-Methode „bewältigt“.

Der Weg von der Aggregatdatenanalyse zur Analyse von Individualdaten ist, grob gesagt, der allgemeine Weg der empirischen Sozialforschung, insbesondere der Wahlforschung gewesen (vgl. Roth, 2008:25f.) Dass er auch innerhalb der Disziplin Geographie eingeschlagen wurde, scheint mir vor allem vom Ergebnis her deutlich: Aus der Selbstverständlichkeit heraus, mit der die Konzeption, Durchführung und Auswertung von Umfragen - inzwischen in Lehrskripte und -bücher zu den Methoden des Faches (etwa Gebhardt, 1993; Meier Kruker und Rauh, 2005; Reuber und Pfaffenbach, 2005) eingegangen und dementsprechend vermutlich auch in Lehrpläne geographischer Institute integriert sind. Die Frage, warum Studierende der Geographie sich Themen und Techniken des Surveys aneignen sollen, ist offensichtlich kein Gegenstand methodischer Fachdiskussionen (mehr).

Inhaltlich betrachtet bedeutete diese Neuorientierung eine Hinwendung auf per Befragung erhebbare individuelle Eigenschaften, Handlungen und Verhaltensweisen sowie auf subjektive Einstellungen und Überzeugungen. Methodisch betrachtet verlangte sie Einführungen in die Fragebogenkonstruktion und -gestaltung, in Verfahren der Stichprobenziehung, in die Konzepte der Prüf- und Teststatistik, die zur Überprüfung und Verallgemeinerung von Stichprobenergebnissen notwendig sind, sowie in die für deren Verständnis notwendigen wahrscheinlichkeitstheoretischen Grundlagen. Nur angerissen werden kann hier, dass diese Reorientierung des methodischen Apparates auf dem Gebiet der Geographie darüber hinaus mit einer Neuformulierung des Interesses an „Raum“ einherging. Folgt man den Entwicklungslinien der Sozialgeographie von Weichhart (2008), ist mit der Betonung der Notwendigkeit einer Analyse von Individualdaten zugleich das Forschungsinteresse auf das subjektive (und per Umfrage erhebbare) Raumerleben und Raummachen der Einzelnen verschoben worden (vgl. Weichhart, 2008:109f.). Im Rahmen dieser „mikroanalytischen“ Wende aber veränderte sich auch die Position von „Raum“ in geographischen Forschungsansätzen; er wurde tendenziell von einer das soziale Handeln und Verhalten erklärenden in eine selbst zu erklärende Variable transformiert.

2. Widerspruch gegenüber dem Nachweis des ökologischen Fehlschlusses und den daraus abgeleiteten methodischen Konsequenzen wurde in verschiedenen Formen von jenen Forschungszweigen artikuliert, die rück- 
blickend in die sozialökologische Tradition der social area analysis bzw. der Sozialraumanalyse gestellt werden können und nach Weichhart (2008:114) als „Raumstrukturforschung" zusammenzufassen sind.

a. In seiner offensiven Variante wurde dieser Widerspruch als Rehabilitation des ökologischen SchlieBens organisiert. Für diese Form der Gegenbewegung steht exemplarisch der bereits genannte Etablierungsversuch einer ,statistischen Geographie“. In Statistical Geography wurde sich der Herausforderung Robinsons insofern direkt angenommen, als dass dort zwei statistische Verfahren präsentiert werden, die in den 1950er Jahren sowohl von Otis und Beverly Duncan selbst als auch von dem Chicagoer Kollegen Leo Goodman in unmittelbarer Reaktion auf Robinsons Kritik entwickelt worden sind: Die „method of bounds“ und die ,ecological regression". Beide Verfahren sind zuerst 1953 ebenfalls in der American Sociological Review vorgestellt worden (vgl. Duncan und Davis, 1953; Goodman, 1953); in Statistical Geography nimmt vor allem die Darstellung letzterer ausgiebig Raum ein (vgl. Duncan et al., 1961:68-80). Beide Verfahren sollen zeigen, dass ein Rückschluss von der Aggregat- auf die Individualebene eben doch möglich ist - mit einer wichtigen, Robinsons Kritik verarbeitenden Einschränkung: Mit ihnen wurde versucht, Zusammenhänge auf der Individualebene über die Analyse von Aggregatdaten einschätzbar zu machen. Es ging mit diesen Verfahren also nicht mehr um die kritikanfällige, direkte Übertragung der Ergebnisse ökologischer Korrelationen auf die Individualebene. Stattdessen ging es um Versuche, aus gegebenen Aggregatdaten heraus eine Bandbreite bzw. einen Möglichkeitsraum der Stärke des $\mathrm{zu}$ vermutenden Zusammenhangs auf Individualebene angebbar zu machen (für eine genauere Vorstellung der unterschiedlichen Logiken der beiden Verfahren siehe z. B. Gschwend, 2006; Freedman, 2001; King, 1997).

Mit dieser Modernisierung des statistischen Apparates erschien das ökologische Schließen als methodische Praxis wieder rehabilitiert, zumindest in den Augen einer sozialökologisch orientierten Forschung (vgl. etwa Hawley, 1962:487) Allerdings vermochten die Verfahren aufgrund ihrer Limitierungen in den folgenden Dekaden offensichtlich nicht, das von Robinsons Problematisierung evozierte Misstrauen gegenüber der Praxis des ökologischen Schließens grundsätzlich aufzuheben. Das ökologische Schließen bleibt auch und gerade vor dem Hintergrund eines anwachsenden Pools an Survey-Daten sowie des Aufbaus umfangreicher Panels, auf die die Scientific Communities seit den 1960er Jahren zunehmend zurückgreifen können, in den folgenden Dekaden als eine bewusst gewählte Forschungsmethode ein randständiges Unterfangen. Insbesondere an der Schnittstelle von politikwissenschaftlicher und geographischer Wahlforschung wurde allerdings systematisch weiterverfolgt, Lösungsangebote für das ökologische Schließen zu entwickeln (siehe für einen Überblick Johnston und Pattie, 2001).

Mit der Mitte der 1990er Jahre von King (1997) präsentierten statistischen Methode liegt heute ein Verfahren für das ökologische Schließen vor, das selbstverständlich in Kings eigenem, aber auch im Urteil eines Großteils der entsprechenden Fachdiskussion - ein weitgehend zuverlässiges Einschätzen von Beziehungen auf der Individualebene aus räumlich erfassten Aggregatdaten heraus erlaubt (vgl. King, 1997; für einen Überblick über die Entwicklungsgeschichte der Konzepte ökologischen Schließens, Kings Lösungsversuch und die daran anhängigen Debatten siehe auch Gschwend, 2006; Withers, 2001).

b. In ihrer defensiven Variante weicht die Widerspruchsorganisation der direkten Konfrontation mit Robinsons Problematisierung aus. Stattdessen wird der Hinweis auf den ökologischen Fehlschluss zunächst akzeptiert, um ihn sodann auf neues Terrain umzulenken und für die Erschließung einer veränderten, komplexeren Forschungspraxis produktiv zu machen. An die Stelle der direkten Abwehr tritt die Kooption der Problematisierung des ökologischen Fehlschlusses zugunsten einer Kombination von Individual- und Aggregatdatenanalyse: die Durchführung einer statistischen Mehrebenenanalyse (multilevel analysis, MLA).

Verfahren der MLA wurden in den englischsprachigen Sozialwissenschaften seit den 1960er, in den entsprechenden deutschsprachigen Wissenschaftszweigen seit Ende der 1960er, Anfang der 1970er Jahre bekannt gemacht. Ihre Einführung ist regelmäßig als Reaktion auf Robinsons Nachweis des ökologischen Fehlschlusses gerechtfertigt worden (vgl. Bahrenberg und Giese, 1990:203f.; Fassmann, 1984:9f.; Kemper, 1983:1f.). Noch heute wird einer der Vorteile der MLA darin gesehen, ,dass sie im Gegensatz zu rein ökologischen Analysen gegen den von Robinson (1950) entdeckten ökologischen Fehlschluss gefeit“ (Langer, 2009:21) sei. Auch Voss (2007:468f.) präsentiert die Mehrebenenanalyse als die adäquate methodische Reaktion auf Robinsons Kritik.

Verfechter der MLA sehen die Konsequenz aus Robinsons Kritik am ökologischen Fehlschluss weder in der Aufgabe noch in der Verteidigung von 
Aggregatdatenanalysen, sondern in einer Erweiterung des Analysehorizonts. Dafür wird zum einen an die schon angedeutete Pluralisierung des Forschungsterrains in Mikroebene und Makroebene(n) angeknüpft, zum anderen auf der Grundannahme von Kontexteffekten aufgebaut. Letztere sollen die Einwirkungen fassbar machen, die wie auch immer spezifizierte Umfelder auf individuelle Einstellungs-, Handlungs- und Verhaltensmuster haben können. Beide Stränge werden zusammengeführt zu der Überzeugung, dass diese Umfelder als die der Individual- bzw. Beobachtungsebene übergeordneten Ebenen operationalisiert werden können. Im Rahmen von zu entwickelnden Mehrebenenmodellen ist sodann der Einfluss der übergeordneten auf die untergeordnete Ebene zu erforschen (vgl. z. B. Kemper, 1983; für eine Darstellung der regressionsanalytischen Grundlagen der MLA siehe etwa Pötschke, 2006).

Mit der Forderung nach Mehrebenenanalysen konnte Robinsons Kritik am ökologischen SchlieBen also neu gerahmt werden. Demnach hatte Robinson mit dem Nachweis des ökologischen Fehlschlusses zu Recht ein Schließen von der (bzw. einer) Makro- auf die Mikroebene verurteilt, weil für verschiedene Untersuchungsebenen unterschiedliche statistische Zusammenhangsbehauptungen festgehalten werden können; allerdings ist er bei diesem Nachweis verblieben. Positiv gewendet aber fordert sein Ergebnis dazu auf, Zusammenhangsbehauptungen nicht nur auf einer, sondern auf verschiedenen Analyseebenen zu verfolgen und kombiniert zu betrachten.

Insofern zudem daran gegangen werden konnte, diejenigen Kontexte subjektiver Einstellungen, individuellen Handelns und Verhaltens, welche „die soziale Umwelt, das kulturelle und soziale Milieu des Individuums beschreiben“ (Bahrenberg und Giese, 1990:203), als sozialräumliche Kontexteffekte aufzufassen, war mit dieser Reaktion auf Robinsons Kritik zugleich eine Wiederaufwertung raumbezogener, auf Aggregatdaten gestützten Analysen verbunden. Mit der Formulierung explizit räumlich verstandener Aggregierungshierarchien - etwa in der „Abfolge Individuum - Haushalt - Nachbarschaft - Wohnquartier - Gemeinde - Region - Staat“" (Kemper, 1983:3) - als die zumindest indirekten Indikatoren für solche Kontexteffekte, die ,durch soziale Gruppen (Familie, Nachbarn) oder sozio-kulturelle Wert- und Normengeflechte" (Kemper, 1983:3) bestimmt würden, eröffnete sich in der Geographie, aber auch etwa in der stadtsoziologischen Forschung (vgl. Friedrichs, 1977:350ff.) erneut eine Möglichkeit, das methodisch-empirische Arbeiten mit Raumaggregatdaten legitim zu halten.

c. Sowohl die offensive als auch die defensive Variante der Widerspruchsorganisation gegen Robinsons Kritik konnte auf eine gemeinsame, feste Rückzugslinie vertrauen, die vermutlich eine Grundhaltung der quantitativ orientierten, raumbezogenen Forschungsansätze in- und außerhalb geographischer Kontexte zum Ausdruck bringt: Die Betonung eines Eigenwerts der (räumlich bezogenen) Aggregatdatenanalyse.

Dieser Eigenwert konnte (und kann) zunächst einmal forschungspragmatisch begründet werden: Angesichts mangelnder alternativer Datenquellen bleibt der Rückgriff auf Aggregatdaten oftmals die einzige Möglichkeit, überhaupt eine grobe Orientierung über vermutete Zusammenhänge zu gewinnen oder erste explorative Schritte in Hinblick auf eine dann folgende Erhebung und Analyse von Individualdaten $\mathrm{zu}$ unternehmen (vgl. King et al., 2004:1). Zudem ließ (und lässt) sich in der Auseinandersetzung mit der Kritik des ökologischen Fehlschlusses dazu übergehen, solche Fragestellungen herauszustreichen, die sich sinnvoll mit Hilfe einer Aggregatdatenanalyse angehen lassen und demnach keine Gefahr laufen, sich in ökologische Fehlschlüsse zu verstricken (vgl. Voss, 2007:464).

Das Beharren auf einem Eigenwert von raumbezogenen Aggregatdatenanalysen war praktisch aber anscheinend vor allem mit der Vorstellung eines arbeitsteiligen Wissenschaftsprozesses zu untermauern und durchzusetzen. In dem Maße, wie die raumbezogene Aggregatdatenanalyse weniger als ein Mittel propagiert wurde, mit dem zu generellen Erklärungen sozialen Handelns und Verhaltens geschritten werden kann, sondern sie mehr als Selbstzweck einer teilbereichsbezogenen Forschung ausgegeben wurde, konnte ihr zugleich die Rolle zugesprochen werden, vor allem beschreibende Aussagen über (sozial-)räumliche Strukturierungen zu leisten sowie Expertise über die relevanten Elemente (sozial-)räumlicher Systeme für eine politisch-administrative Gestaltung der Raumorganisation aufzubieten. ${ }^{4}$ Subjektive Einstellungen sowie individuelles Handeln und Verhalten in den Blick zu nehmen sowie übergreifende Erklärungen sozialer Wirklichkeit aufzubieten ist demnach auf anderen Wissenschaftsfeldern zu leisten.

\footnotetext{
${ }^{4}$ So jedenfalls lässt sich etwa die von Gerhard Bahrenberg Ende der 1970er Jahre unter der Überschrift Von der Anthropogeographie zu Regionalforschung (1979) gezogene Zwischenbilanz zur Entwicklung der deutschsprachigen quantitativ orientierten Geographie lesen.
} 
Methodisch gestützt wurde diese Rückzugsbewegung auf einen klarer definierten, weil in seiner Aufgabenreichweite beschränkten Einsatz der Raumaggregatdatenanalyse seit den 1960er Jahren, verstärkt seit Anfang der 1970er Jahre mit den Verfahren der Faktoren- und der Clusteranalyse. Diese Verfahren erlauben die Reduktion von Beziehungen in einem umfangreichen Datenmaterial auf wenige, aussagekräftige Variablen und die Klassifizierung von vielfältigen Merkmalskombinationen. In den raumbezogen arbeitenden Sozialwissenschaften wird mit ihrer Hilfe zur Typisierung von räumlichen Beobachtungseinheiten bzw. zu „, Regionalisierungen" geschritten (vgl. Sedlacek, 1978; Söker, 1977:120ff.).

Dabei kommt dem Einsatz der Faktoren- und Clusteranalyse vorwiegend als das methodische Mittel zur Datenreduktion und Klassifizierung ein theorieferner $\mathrm{Zug} \mathrm{zu}$; mit ihnen durchgeführte Analysen fallen mehr beschreibend oder explorativ denn als prüfend oder erklärend aus (vgl. Sedlacek, 1978:19). Die von Robinson am ökologischen Fehlschluss vorgenommene Problematisierung der Aussagekraft von Beziehungen zwischen räumlich erfassten Beobachtungen für ein erklärendes Verständnis der sozialen Wirklichkeit konnte so zumindest vordergründig umgangen, der Hinterfragung der Legitimität einer methodisch-empirisch auf Raumaggregatdaten sich stützenden Forschung ausgewichen werden.

\section{Fazit}

„Revolutionäre produzieren Konterrevolutionäre“ (Galtung, 1973:128), bemerkte Johan Galtung in seinen Überlegungen zu Eine strukturelle Theorie der Revolution (1973). Diese „simple Dialektik“ (Galtung, 1973:128) lässt sich auch für eine Betrachtung derjenigen Epoche geographischer Wissensproduktion fruchtbar machen, die regelmäßig als $\mathrm{qR}_{\mathrm{Geo}} \mathrm{zu}-$ sammengefasst wird.

In diesem Beitrag wurde verdeutlicht, dass und wie der Nachweis des ökologischen Fehlschlusses durch Robinson (1950) einer disziplinübergreifenden bzw. -diffundierenden Wissenschaftspraxis, nämlich dem methodisch-empirischen Arbeiten mit räumlich gebundenen Aggregatdaten, ihre legitimatorische Basis und ihren anwendungspraktischen Boden entzogen hatte. Zudem wurde an den Aufstieg der Survey-Methode zur neuen methodischen Basis sozialwissenschaftlicher Untersuchungen erinnert. Unter anderem um dieser Revolution der sozialwissenschaftlichen Wissenschaftspraxis begegnen und ihre wissenschaftspraktischen Folgen verarbeiten zu können, wurde auf dem Feld der Geographie daran gegangen, den methodisch-statistischen Apparat zu modernisieren. Mit diesen Modernisierungsschritten gelang zum einen der Anschluss an die Themen und Techniken der SurveyMethode. Zum anderen umfasste die methodisch-statistische Modernisierung die Entwicklung von oder den Anschluss an Innovationen auf den Gebieten des ökologischen Schließens, der statistischen Mehrebenenanalyse sowie der Faktorenund Clusteranalyse. Auf diesen Wegen gelang es zudem, mit Raumaggregatdaten operierende Forschungsansätze in der Erforschung der sozialen Wirklichkeit wissenschaftlich legitim zu halten und das in ihnen korporierte wissenschaftliche Kapital (siehe dazu etwa Bourdieu, 1998:31ff.) gegen eine drohende Entwertung zu behaupten.

In (Selbst-)Darstellungen der $\mathrm{qR}_{\mathrm{Geo}}$ wie die von Burton (1970) und dem damit tradierten Rechtfertigungszusammenhang allerdings wirken diese reaktiven Züge vergessen, die Momente der Anpassung an bzw. der Abwehr von Kritik verdrängt. Stattdessen erscheint die $\mathrm{qR}_{\mathrm{Geo}}$ genannte Restrukturierung von Wissensbeständen und Wissenschaftspraktiken zu einem Unterfangen rationalisiert, das ausschließlich aus einer Kritik an einer überkommenen Wissenschaftstradition und deren Defiziten heraus motiviert worden ist. Im tradierten Rechtfertigungszusammenhang der $\mathrm{qR}_{\mathrm{Geo}}$ wird die damit benannte Neustrukturierung geographischer Wissensproduktion als ein Ereignis betrachtet, das zwar vielleicht von geographieexternen Einflussfaktoren mitbestimmt wurde, zum Beispiel von anderen Wissenschaftsgebieten angestoßen und von den Karrierewünschen einer nachwachsenden Wissenschaftsgeneration forciert (so schon Burton, 1970) oder von der Entwicklung elektronischer Datenverarbeitung begünstigt (so schon Hägerstrand, 1970) worden ist. Innerhalb solcher Rahmungen aber wird die $\mathrm{qR}_{\mathrm{Geo}}$ vor allem als der epistemische Bruch mit einer traditionellen, unwissenschaftlich eingeschätzten Geographie erinnert. Es bleiben somit solche Aspekte aus der Betrachtung ausgeklammert, an denen Kontinuitäten über den Prozess der Revolutionierung hinweg deutlich werden (vgl. Michel, 2014) oder die die $\mathrm{qR}_{\mathrm{Geo}} \mathrm{mehr}$ als Abwehrbemühung denn als Neuerung, mehr als Restauration denn als Revolution erscheinen lassen.

In dieser Vereinseitigung der Frontstellungen wiederum liegen, so darf vermutet werden, einerseits die Erfolgsbedingungen des quantitativ orientierten Modernisierungsprozesses in der Geographie. Erst die Engführung der (Selbst-)Darstellung der $\mathrm{qR}_{\mathrm{Geo}}$ auf eine Absatzbewegung gegen eine rückständige Wissenschaftstradition ermöglichte es, tatsächliche Leistungen und Erfolge der quantitativ ausgerichteten Neuorientierung auch als wissenschaftlichen Fortschritt zur Geltung bringen zu können. Zugleich aber mutet diese Vereinseitigung in doppelter Weise problematisch an: Zum einen macht sie das Problem des ökologischen Fehlschlusses vergessen. Der ökologische Fehlschluss repräsentiert in nunce ein Erklärungsmodell sozialer Wirklichkeit, das diese von ihren Verräumlichungen selbst her bestimmt sieht; sein Nachweis war ein Stück (Selbst-)Aufklärung der Sozialwissenschaften über diese Fehlleistung. Den provokativen Charakter der Kritik des ökologischen Fehlschlusses 
über deren Ausklammerung aus der Geschichte der Quantifizierung der Geographie vergessen zu machen, trägt zur Persistenz dieser „Raumfalle“ (Lippuner und Lossau, 2004) bei. Zum anderen aber lässt sie die methodisch-statistischen Lernprozesse in- und außerhalb der Geographie unbeachtet, die in den letzten Jahrzehnten im Umgang mit dem Problem des ökologischen Fehlschlusses gemacht wurden.

Datenverfüg barkeit. Für diesen Artikel wurden keine Datensätze genutzt.

Interessenkonflikt. Der Autor erklärt, dass kein Interessenkonflikt besteht.

Danksagung. Der Autor dankt den Teilnehmerinnen und Teilnehmern des Workshops „Raum. Gesetze. Daten.“(2015) in Erlangen für die Diskussion erster Thesen zu diesem Beitrag.

Edited by: Benedikt Korf

Reviewed by: three anonymous referees

\section{Literatur}

Achen, C. H. und Shively, W. P.: Cross-Level Inference, Univ. of Chicago Press, Chicago, 1995.

Bahrenberg, G.: Von der Anthropogeographie zu Regionalforschung: Eine Zwischenbilanz, in: Zur Situation der deutschen Geographie zehn Jahre nach Kiel, Herausgeber: Sedlacek, P., Osnabrücker Studien zur Geographie, Bd. 2, Fachbereich 2 der Universität Osnabrück, Osnabrück, 59-68, 1979.

Bahrenberg, G. und Giese, E.: Statistische Methoden in der Geographie, 3. überarb. Aufl., Teubner, Stuttgart, 1990.

Barnes, T. J.: A history of regression: actors, networks, machines, and numbers, Environ. Plan. A, 30, 203-223, https://doi.org/10.1068/a300203, 1998.

Barnes, T. J. und Hannah, M.: The place of numbers: histories, geographies, and theories of quantification, Environ. Plan. D, 19, 379-383, https://doi.org/10.1068/d1904ed, 2001.

Bartels, D.: Theoretische Geographie: Zu neuerer englischsprachiger Literatur, Geogr. Z., 57, 132-144, 1969.

Bartels, D. (Hrsg.): Wirtschafts- und Sozialgeographie, Kiepenheuer \& Witsch, Köln, Berlin, 1970.

Bourdieu, P.: Vom Gebrauch der Wissenschaft: Für eine klinische Soziologie des wissenschaftlichen Feldes, UVK, Konstanz, 1998.

Burton, I.: The quantitative revolution and theoretical geography, The Canadian Geographer, 7, 151-162, 1963.

Burton, I.: Quantitative Revolution und theoretische Geographie, in: Wirtschafts- und Sozialgeographie, Herausgeber: Bartels, D., Kiepenheuer \& Witsch, Köln, Berlin, 95-109, 1970.

Calhoun, C. und VanAntwerpen, J.: Orthodoxy, heterodoxy, and hierarchy: "mainstream" sociology and its challengers, in: Sociology in America: A History, Herausgeber: Calhoun, C., Univ. of Chicago Press, Chicago, 367-410, 2007.
Duncan, O. D. und Davis, B.: An alternative to ecological correlation, Am. Soc. Rev., 18, 665-666, 1953.

Duncan, O. D., Cuzzort, R. P. und Duncan, B.: Statistical Geography: Problems in Analyzing Areal Data, The Free Press of Glencoe, Glencoe, Ill., 1961.

Fassmann, H.: Mehrebenenanalyse - Fehlschlußproblem - Aggregierungsverzerrung: Ein methodischer Beitrag zur Analyse komplexer Datensätze in der Geographie, Geographischer Jahresbericht aus Österreich, 41, 7-26, 1984.

Firebaugh, G.: Ecological fallacy, statistics of, in: International Encyclopedia of the Social \& Behavioral Sciences, Herausgeber: Smelser, N. J. und Baltes, P. B., Elsevier, Oxford, 4023-4026, 2001.

Freedman, D. A.: Ecological inference, in: International Encyclopedia of the Social \& Behavioral Sciences, Herausgeber: Smelser, N. J. und Baltes, P. B., Elsevier, Oxford, 4027-4030, 2001.

Friedrichs, J.: Stadtanalyse: Soziale und räumliche Organisation der Gesellschaft, Rowohlt, Reinbek bei Hamburg, 1977.

Galtung, J.: Eine strukturelle Theorie der Revolution, in: Herrschaft und Krise: Beiträge zur politikwissenschaftlichen Krisenforschung, Herausgeber: Jänicke, M., Westdeutscher Verlag, Opladen, 121-167, 1973.

Gebhardt, H.: Forschungsmethoden in der Kulturgeographie, Kleinere Arbeiten aus dem Geographischen Institut der Universität Tübingen, 13, Selbstverl. d. Geograph. Inst. d. Univ. Tübingen, Tübingen, 1993.

Giese, E.: Vorwort, in: Symposium „Quantitative Geographie“: Möglichkeiten und Grenzen der Anwendung mathematischstatistischer Methoden in der Geographie, Herausgeber: Giese, E., Selbstverl. d. Geograph. Inst. d. Univ. Gießen, Gießen, 5-8, 1975.

Goodman, L. A.: Ecological regressions and behavior of individuals, Am. Soc. Rev., 18, 663-664, 1953.

Gschwend, T.: Ökologische Inferenz, in: Methoden der Politikwissenschaft: Neuere qualitative und quantitative Analyseverfahren, Herausgeber: Behnke, J., Gschwend, T., Schindler, D. und Schnapp, K.-U., Nomos, Baden-Baden, 227-237, 2006.

Haas, G.: Fehlschluß, in: Enzyklopädie Philosophie und Wissenschaftstheorie, Herausgeber: Mittelstraß, J., WBG, Darmstadt, 634-635, 2013.

Habermas, J.: Theorie des kommunikativen Handelns, Band 1: Handlungsrationalität und gesellschaftliche Rationalisierung, 2. Aufl., Suhrkamp, Frankfurt am Main, 1982.

Hägerstrand, T.: Der Computer und der Geograph, in: Wirtschaftsund Sozialgeographie, Herausgeber: Bartels, D., Kiepenheuer \& Witsch, Köln, Berlin, 278-300, 1970.

Hawley, A. H.: Theorie und Forschung in der Sozialökologie, in: Handbuch der Empirischen Sozialforschung, Band 1, Herausgeber: König, R., Enke, Stuttgart, 480-497, 1962.

Hirschman, A. O.: Exit, Voice, and Loyalty: Responses to Decline in Firms, Organizations, and States, Harvard Univ. Press, Cambridge, Mass., 1970.

Hirschman, A. O.: Abwanderung und Widerspruch: Reaktionen auf Leistungsabfall bei Unternehmungen, Organisationen und Staaten, Mohr, Tübingen, 1974.

Johnston, R. und Pattie, C.: On Geographers and Ecological Inference, Ann. Assoc. Am. Geogr., 91, 281-282, https://doi.org/10.1111/0004-5608.00243, 2001. 
Kemper, F.-J.: Zur Notwendigkeit der Berücksichtigung regionaler Kontexteffekte bei sozialwissenschaftlichen Analysen, Bundesforschungsanstalt für Landeskunde und Raumordnung, Bonn, 1983.

King, G.: A Solution to the Ecological Inference Problem: Reconstructing Individual Behavior from Aggregate Data, Princeton Univ. Press, Princeton, NJ, 1997.

King, G., Rosen, O. und Tanner, M. A.: Information in Ecological Inference: An Introduction, in: Ecological Inference: New Methodological Strategies, Herausgeber: King, G., Rosen, O. und Tanner, M. A., Cambridge Univ. Press, Cambridge, UK, New York, 1-12, 2004.

Kuhn, T. S.: The Structure of Scientific Revolutions, Univ. of Chicago Press, Chicago, 1962.

Kuhn, T. S.: Die historische Struktur wissenschaftlicher Entdeckungen: Studien zur Struktur der Wissenschaftsgeschichte, Suhrkamp, Frankfurt am Main, 1978.

Kuhn, T. S.: Die Struktur wissenschaftlicher Revolutionen, 18. Aufl. von der 2. rev. Aufl., um das Postskriptum von 1969 erg. Aufl., Suhrkamp, Frankfurt am Main, 2003.

Langer, W.: Mehrebenenanalyse: Eine Einführung für Forschung und Praxis, 2. Aufl., Verl. für Sozialwiss., Wiesbaden, 2009.

Lippuner, R. und Lossau, J.: In der Raumfalle: Eine Kritik des spatial turn in den Sozialwissenschaften, in: Soziale Räume und kulturelle Praktiken, Herausgeber: Mein, G. und Rieger-Ladich, M., transcript, Bielefeld, 47-63, 2004.

McCarty, H. H., Hook, J. C. und Knos, D. S.: The Measurement of Association in Industrial Geography, Department of Geography, State Univ. of Iowa, Iowa City, 1956.

Meier Kruker, V. und Rauh, J.: Arbeitsmethoden der Humangeographie, WBG, Darmstadt, 2005.

Michel, B.: Wir sind nie revolutionär gewesen - Zum Mythos des Kieler Geographentags als der Geburtsstunde einer neuen Geographie, Geogr. Helv., 69, 301-303, https://doi.org/10.5194/gh69-301-2014, 2014.

Oakes, J. M.: Commentary: Individual, ecological and multilevel fallacies, Int. J. Epidemiol., 38, 361-368, https://doi.org/10.1093/ije/dyn356, 2009.

Pötschke, M.: Merhebenenanalyse, in: Methoden der Politikwissenschaft: Neuere qualitative und quantitative Analyseverfahren, Herausgeber: Behnke, J., Gschwend, T., Schindler, D. und Schnapp, K.-U., Nomos, Baden-Baden, 167-179, 2006.

Reuber, P. und Pfaffenbach, C.: Methoden der empirischen Humangeographie: Beobachtung und Befragung, Westermann, Braunschweig, 2005.

Robinson, W. S.: Ecological correlations and the behavior of individuals, Am. Soc. Rev., 15, 351-357, 1950.

Robinson, W. S.: Ecological correlations and the behavior of individuals, Int. J. Epidemiol., 38, 337-341, https://doi.org/10.1093/ije/dyn357, 2009.

Roth, D.: Empirische Wahlforschung: Ursprung, Theorien, Instrumente und Methoden, 2. Aufl., Verl. für Sozialwiss., Wiesbaden, 2008.
Schäfer, A., Vehrkamp, R. und Gagné, J. F.: Prekäre Wahlen: Milieus und soziale Selektivität der Wahlbeteiligung bei der Bundestagswahl 2013, Bertelsmann Stiftung, Gütersloh, 2013.

Schnapp, K.-U., Schindler, D., Gschwend, T. und Behnke, J.: Qualitative und quantitative Zugänge: Eine integrative Perspektive, in: Methoden der Politikwissenschaft: Neuere qualitative und quantitative Analyseverfahren, Herausgeber: Behnke, J., Gschwend, T., Schindler, D. und Schnapp, K.-U., Nomos, Baden-Baden, 1126, 2006.

Sedlacek, P.: Einleitung, in: Regionalisierungsverfahren, Herausgeber: Sedlacek, P., WBG, Darmstadt, 1-19, 1978.

Shaw, C. R., McKay, H. D., Zorbaugh, H. und Cotrell, L. S.: Delinquency Areas: A Study of the Geographic Distribution of School Truants, Juvenile Delinquents, and Adult Offenders in Chicago, Univ. of Chicago Press, Chicago, 1929.

Sheppard, E.: Quantitative geography: representations, practices, and possibilities, Environ. Plan. D, 19, 535-554, https://doi.org/10.1068/d307, 2001.

Sheppard, E.: We have never been positivist, Urban Geography, 35, 636-644, https://doi.org/10.1080/02723638.2014.916907, 2014.

Söker, E.: Das Regionalisierungskonzept: Instrumente und Verfahren der Regionalisierung, Mitteilungen der Geographischen Gesellschaft in Hamburg, 67, Geograph. Ges., Hamburg, 1977.

Subramanian, S. V., Jones, K., Kaddour, A. und Krieger, N.: Revisiting Robinson: the perils of individualistic and ecologic fallacy, Int. J. Epidemiol., 38, 342-360, https://doi.org/10.1093/ije/dyn359, 2009.

te Grotenhuis, M., Eisinga, R. und Subramanian, S. V.: Robinson's Ecological correlations and the behavior of individuals: methodological corrections, Int. J. Epidemiol., 40, 1-3, https://doi.org/10.1093/ije/dyr081, 2011.

Thomas, E. N. und Anderson, D. L.: Additional comments on weighting values in correlation analysis of areal data, Ann. Assoc. Am. Geogr., 55, 492-505, 1965.

Voss, P. R.: Demography as a Spatial Social Science, Popul. Res. Policy Rev., 26, 457-476, https://doi.org/10.1007/s11113-0079047-4, 2007.

Weichhart, P.: Entwicklungslinien der Sozialgeographie: Von Hans Bobek bis Benno Werlen, Steiner, Stuttgart, 2008.

Weichhart, P.: Der Kieler Geographentag 1969 - Modernisierungsschub, Mythos, Paradigmenwandel oder vergessene Geschichte?, Geogr. Helv., 71, 7-13, https://doi.org/10.5194/gh-71-7-2016, 2016.

Weingart, P.: Wissensproduktion und soziale Struktur, Suhrkamp, Frankfurt am Main, 1976.

Weingart, P.: Wissenschaftssoziologie, transcript, Bielefeld, 2003.

Withers, S. D.: Quantitative methods: advancement in ecological inference, Prog. Human Geogr., 25, 87-96, 2001. 